\title{
Données nouvelles sur la morphologie et la biologie de Parvatrema timondavidi Bartoli 1963 (Trematoda: Digenea).
}

\author{
par Pierre BARTOLI
}

Depuis longtemps déjà, les Moules sont connues par leurs formations perlières. Selon A. Giard (1907), O. Worm les signalait au milieu du XVI' siècle sur les côtes danoises, à Roeskild, près de Copenhague. Mais il fallut attendre un peu plus de deux siècles pour découvrir quel était l'agent margaritigène. C'est R. Garner qui, en 1861, reconnut qu'il s'agissait là d'un Distome.

En France, le Baron d'Hamonville (1894) signale à Billiers (Morbihan) un banc de Mytilus edulis L. renfermant des perles en grande quantité. Dans les Moules de ce même banc, R. Dubois (1901) retrouva le distome signalé par R. Garner en Angleterre et proposa le nom de Distomum margaritarum. L'année suivante et toujours à Billiers, H.-L. Jameson (1902) retrouva $D$. margaritarum qu'il considéra comme le stade larvaire de Distomum (Brachycoelium Duj. $1845=$ Lecithodendrium Looss 1896) somateriae Levinsen 1892. En 1903, R. Dubois étudiant les M. galloprovincialis Lmck du littoral des Bouches-du-Rhône (France), y trouva « non seulement une autre espèce, mais encore un animal dont les conditions de milieu sont très différentes ». En 1907, modifiant son opinion première, il admit que le distome de $M$. edulis et celui de M. galloprovincialis appartenaient à la même espèce. Reprenant ce problème, R.-Ph. Dollfus (1923) s'aperçut que la métacercaire hébergée par $M$. galloprovincialis représentait bien en fait une espèce différente de celle rencontrée dans $M$. edulis; l'ayant reconnue dans une figure donnée par R. Dubois (1909), il la nomma Gymnophallus duboisi.

Contestant la validité de cette dernière espèce, A. Palombi (1924) la mit en synonymie avec G. margaritarum, mais plus tard (1934) il revint sur cette affirmation.

En 1911, D. T. Sinitzine décrit Adolescaria perla de $M$. edulis et de Venus sp. de la Mer Noire.

En 1940, A. Palombi trouve dans le manteau de $M$. galloprovincialis du Golfe de Tarente (Italie) une métacercaire margaritigène : Gymnophallus perligena.

En 1958, H. W. Stunkard et J. R. Uzmann décrivent Metacercaria II des $M$. edulis de la région de Newburyport (Massachusetts). 

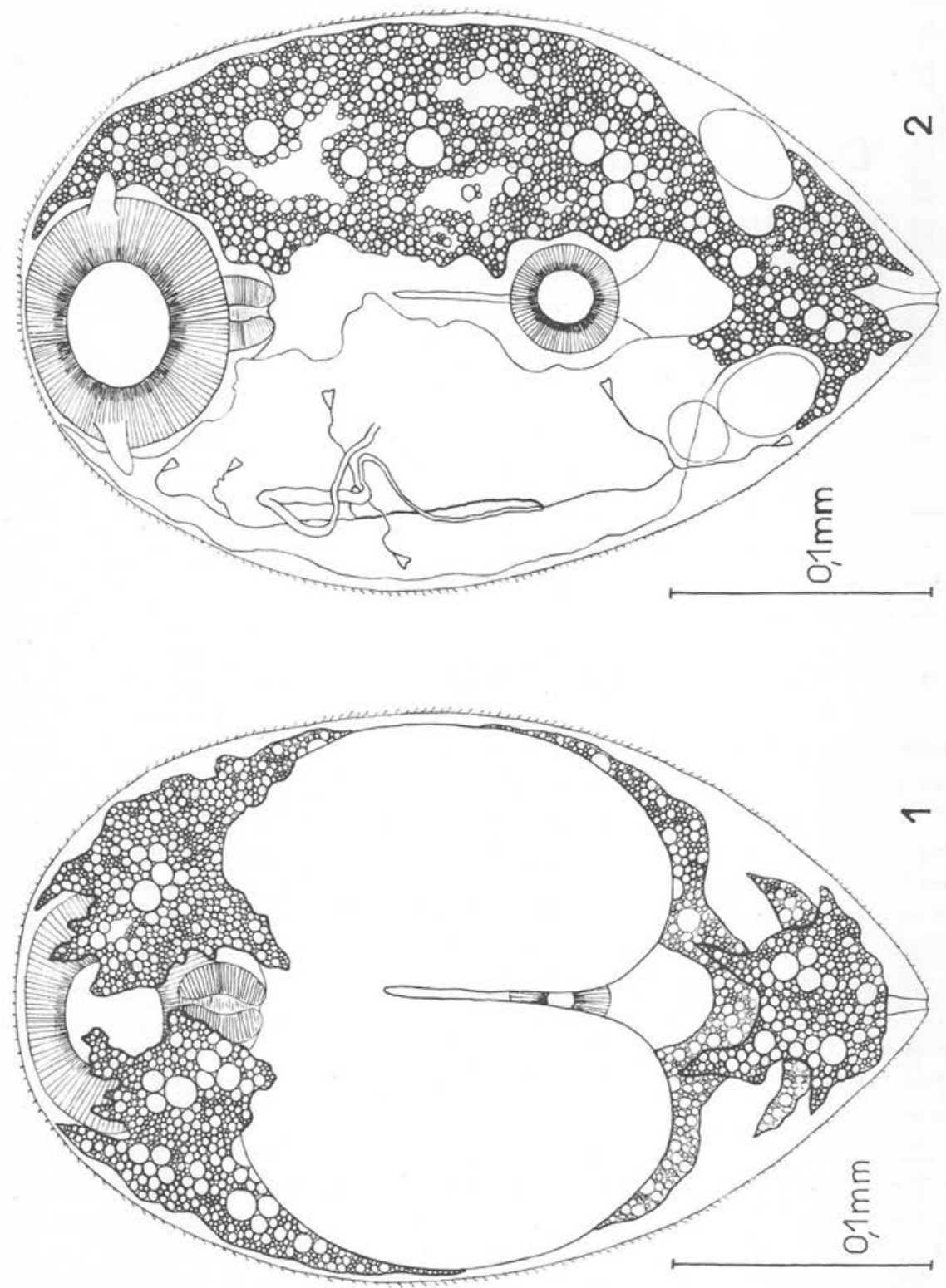

FIG. 1. - Parvatrema timondavidi Bartoli, 1963. Métacercaire en vue dorsale

Fig. 2. - Parvatrema timondavidi Bartoli, 1963. Appareil excréteur de la métacercaire (vue ventrale) 
En 1962, L. Szidat découvre dans $M$. platensis des côtes d'Argentine (Puerto Quequén) des métacercaires libres et enkystées appartenant à une nouvelle espèce : G. australis. La même année, E. A. Zelikman trouva dans les $M$. edulis de la Baie de Kandalachka (Mer Blanche) des métacercaires appartenant à G. choledochus Odhner 1900 alors que, selon l'auteur et d'après Belopolskaïa, sur la côte mourmane orientale, les métacercaires hébergées par ces mêmes lamellibranches seraient le stade larvaire de G. somateriae Odhner 1900 .

Toutes ces formes larvaires parasitant les Moules se rattachent au genre Gymnophallus. Jusqu'à ce jour, une seule espèce du genre Parvatrema, très voisin du genre Gymnophallus, y a été rencontrée: P. timondavidi mihi 1963, de M. galloprovincialis du Golfe de Marseille (France).

Je me propose d'apporter ici des données nouvelles non seulement sur l'adulte obtenu expérimentalement, mais aussi sur la métarcercaire, notamment en ce qui concerne son appareil génital dont il faut noter d'ores et déjà l'état de maturation avancé,

\section{La Métacercaire (fig. 1 à 4)}

\section{LOCALISATION :}

Dans le manteau, mais beaucoup plus souvent encore dans le rachis des branchies, sur la ligne où celles-ci s'insèrent sur le manteau.

FIG. 3. - Parvatrema timondavidi Bartoli, 1963. Région antérieure de la métacercaire montrant les glandes céphaliques (vue dorsale)

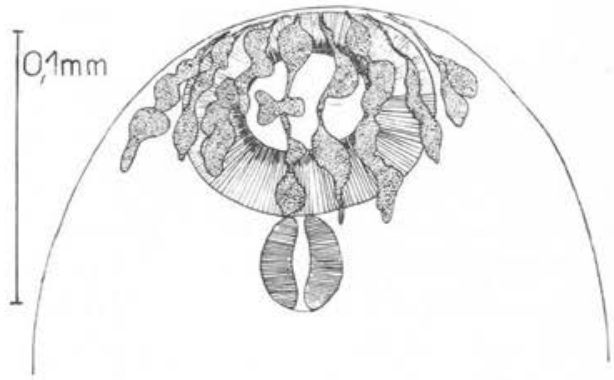

\section{CORPS :}

La forme générale est celle d'un losange arrondi à l'extrémité antérieure et pointu à l'extrémité postérieure. Coloration jaunâtre. Toute la surface de la cuticule est ornée d'épines disposées en quinconce. Il n'y a pas de kyste métacercarien proprement dit mais on observe souvent et non toujours une enveloppe muqueuse où se reconnaît une délicate striation rayonnante. Ce sac muqueux constitue un centre d'attraction pour les particules calcaires qui finiront par circonscrire totalement la métacercaire, engendrant ainsi une perle.

Dimensions : $310-348 \mu \times 148-256 \mu$. 
Ventouse orale subterminale assez volumineuse : 75-81 $\mu \times 90-92 \mu$. Elle présente latéroventralement une paire d'auricules ( ear-like projections ») faisant fortement saillie. Quatre paires de glandes céphaliques (fig. 3) couvrent dorsalement la région de la ventouse orale; leurs canaux excréteurs débouchent en avant de celle-ci.

La ventouse ventrale $(29-37 \mu \times 36-46 \mu)$ couvre la fin du tiers médian.

Appareil digestif.

La bouche s'ouvre au centre de la ventouse orale.

Il n'y a pas de prépharynx.

Le pharynx musculeux est volumineux : 27-36 $\mu \times 25-32 \mu$.

Esophage absent.

Caecums : volumineux, s'affrontant sur la ligne sagittale; ils couvrent la totalité du tiers médian et peuvent déborder même sur les tiers adjacents. Leur limite postérieure dépasse donc souvent la ventouse ventrale. Ils sont remplis de débris de branchies ou de manteau, ce qui contribue à leur donner une coloration jaunâtre.

ApPareil géNItAl MALE.

Les testicules, de forme ellipsoïdale, sont situés en arrière de la ventouse ventrale, leur grand axe étant parallèle à la paroi latérale du corps. Dimensions : 41-75 $\mu \times 25-35 \mu$. Les spermiductes fusionnent entre eux à la base d'une vésicule séminale indivise, aux parois constituées de cellules turgescentes. Le canal éjaculateur débouche dans l'atrium génital. Malgré le grand nombre d'observations, je n'ai pas réussi à voir s'il y avait des cellules prostatiques.

APPAREIL GÉNITAL FEMELLE.

L'ovaire est subcirculaire; diamètre : 25-35 $\mu$. Il est situé tantôt à droite, tantôt à gauche. L'oviducte, assez long, débouche dans une chambre bipartite ; la partie postérieure (ootype) est ciliée. Canal de Laurer présent. Les cellules vitellines sont groupées en une masse unique, située tantôt à droite, tantôt à gauche, selon que l'ovaire est à gauche ou à droite. L'utérus décrit quelques boucles; le métraterme est peu individualisé ; il débouche à la base de l'atrium génital.

\section{L'ATRIUM GÉNITAL.}

Profond et en forme d'outre, il est enveloppé de cellules difficiles à observer qui, de par leur position, ne peuvent être assimilées à des cellules prostatiques. Cet atrium génital s'ouvre sur la face ventrale, à une distance du bord antérieur de la ventouse ventrale égale au diamètre de celle-ci.

\section{APPAREIL EXCRÉteur.}

Les néphridies, dont la position est remarquablement constante, sont au nombre de six paires ; elles sont disposées selon la formule caractéristique du genre Parvatrema : 


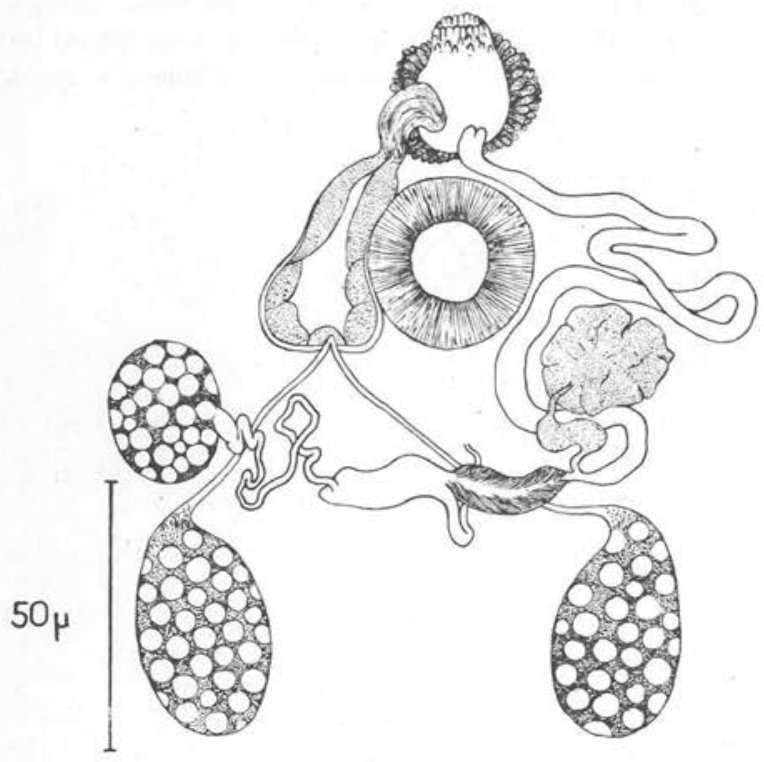

Fig. 4. - Parvatrema timondavidi Bartoli, 1963, Appareil génital de la métacercaire (vue ventrale)

$2[(2+2)+2]=12$. Le canal collecteur terminal est cilié et, après avoir décrit une boucle, pénètre dans la vessie à mi-chemin entre le pharynx et la ventouse ventrale. $\mathrm{La}$ vessie, en forme de $\mathrm{V}$, est remplie de très nombreuses granulations sphériques, brunes et de tailles variables. La branche impaire émet une expansion sur la face dorsale et deux diverticules latéraux. Les branches paires, rejetées sur la face ventrale dans leur parcours moyen du fait du grand développement des caecums, s'étendent jusqu'à l'extrémité antérieure où elles reviennent alors en position dorsale.

\section{L'Adulte (fig. 5)}

Toutes les larves examinées se sont toujours montrées dans le même état avancé de maturation.

J'ai à plusieurs reprises obtenu le développement expérimental de ces métacercaires.

Le 9 juillet 1963, un caneton est infesté, puis autopsié cinq jours après; j’ai retrouvé dans l'intestin moyen cinq sujets adultes, mais morts.

Le 18 septembre 1963, un jeune poussin est disséqué 18 heures après le repas infestant ; j'ai pu retrouver dans l'intestin moyen un individu vivant, mais immature.

Le $1^{\text {er }}$ juin 1964, un jeune Larus argentatus michaellis Naumann est contaminé, puis tué 48 heures après; 14 sujets vivants, montrant tous quelques œufs, sont retrouvés dans le troisième quart de l'intestin. 
Le 11 juin 1964, un autre jeune goéland de la même espèce est tué 88 heures après sa contamination. 53 individus vivants et parfaitement mûrs sont retrouvés. Leur répartition le long de l'intestin était la suivante : $2^{e}$ quart $: 2$ individus ; $3^{e}$ quart $: 32$ individus ; $4^{e}$ quart : 19 individus.

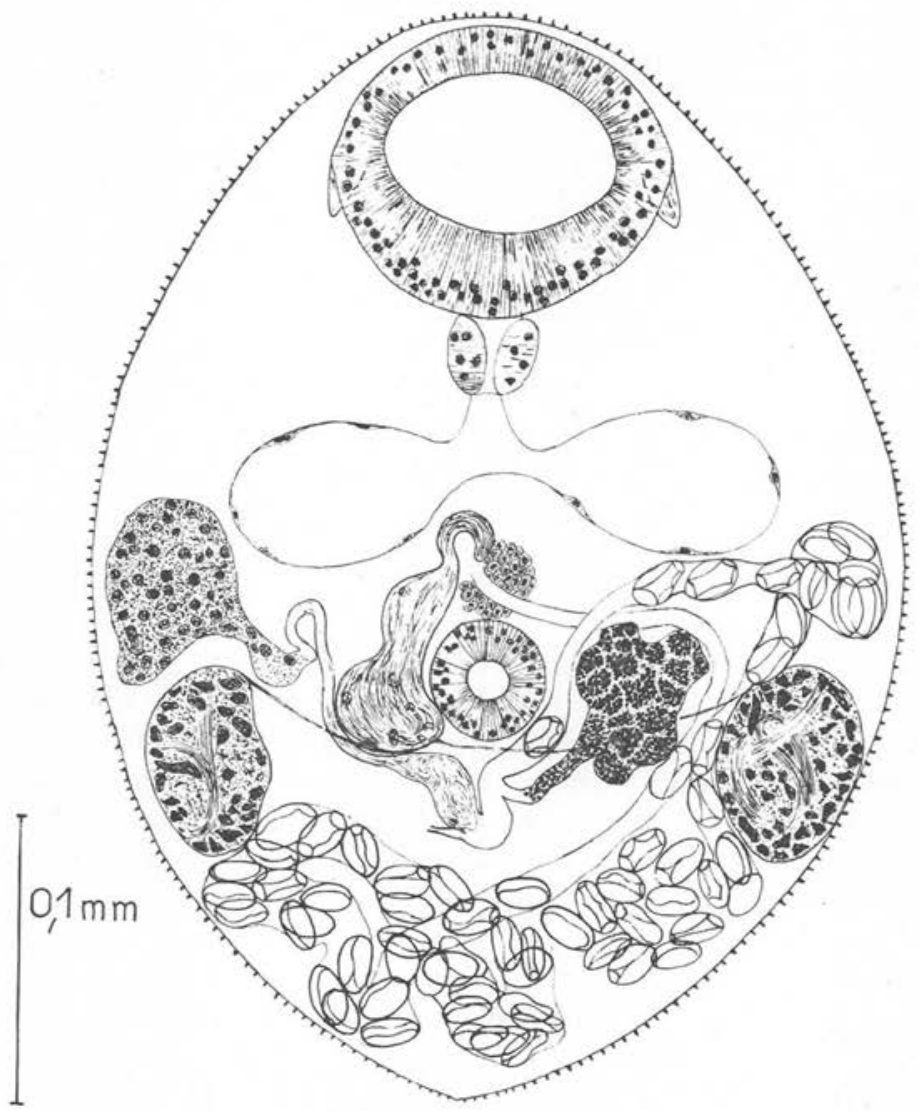

Fig. 5. - Parvatrema timondavidi Bartoli, 1963. Adulte obtenu expérimentalement chez Larus argentatus michaellis, Naumann (vue dorsale)

Les principales modifications subies au cours de cette maturation ont lieu au niveau de la taille de l'animal, de son tube digestif et de son appareil génital.

Les dimensions des individus colorés et montés entre lame et lamelle sont: 330-670 $\mu \times 231-317 \mu$. La ventouse orale $(79-102 \mu \times 96-125 \mu)$ présente la paire d'auricules de la métacercaire. La ventouse ventrale $(36-46 \mu \times 40-46 \mu)$ est située à la fin du tiers médian. Le rapport de la longeur du corps sur celle de la ventouse orale varie entre 3,5 et 5 ; le rapport ventousaire est compris entre 2,2 et 2,6 . Le pharynx (20-36 $\mu \times 25-40 \mu)$ est beaucoup moins compact. Les caecums présentent une direction transversale ; leur taille diminuant beaucoup, il apparaît un œsophage. 
La presque totalité de l'appareil génital est contenue dans la seconde moitié du corps. Les testicules $(56-82 \mu \times 40-69 \mu)$ sont légèrement repoussés vers l'avant du fait du grand développement de l'utérus dans la région post-acétabulaire. La vésicule séminale s'accroît ; sa longueur varie de $60 \mu$ à $76 \mu$.

L'ovaire est aussi repoussé vers l'avant ; ses dimensions sont : 36-40 $\mu \times 20-60 \mu$. Je n'ai pas observé de réceptacle séminal, mais cet organe semble remplacé dans ses fonctions par la chambre située à la fin de l'oviducte. L'utérus décrit plusieurs circonvolutions dont la première est toujours située en avant du testicule opposé à l'ovaire. Les œufs mesurent : 18-22 $\mu \times 9-14 \mu$. Les cellules vitellines sont groupées en une seule masse, entre l'acétabulum et le testicule opposé à l'ovaire.

Le nombre et la position des néphridies demeurent inchangés. La vessie ne varie pas dans sa forme générale, mais les granulations s'éclaircissent et deviennent plus rares.

\section{Discussion}

Parvatrema timondavidi est la seule espèce de ce genre à avoir été signalée dans les Moules. Elle se différencie aisément des métacercaires du genre Gymnophallus parasitant ces lamellibranches par la présence d'auricules à la ventouse orale et par son rapport ventousaire. Ce dernier est cependant assez proche de celui de G. duboisi et de celui de $G$. australis. Ces deux dernières espèces sont en fait de taille plus restreinte $(23-27 \mu \times 12-17 \mu$ pour $G$. duboisi et $23 \mu$ de longueur pour G. australis) et les branches de la vessie ne possèdent pas de diverticules. En outre, chez G. duboisi, l'orifice génital est situé sur la bordure antérieure de la ventouse ventrale.

En dehors de la métacercaire de $P$. timondavidi, on connaît six de ces formes se rapportant au genre Parvatrema: $P$. borinqueñae Cable 1953, P. borealis Stunkard et Uzmann 1958, P. donacis Hopkins 1958, P. homoeotecnum James 1964, P. sp. 1 et $P$. sp. 2, Rebecq 1964. A l'exception de $P$. donacis, l'espèce provençale se distingue facilement des autres par sa grande taille, les rapports ventousaires différents, l'absence d'œsophage, la grande taille des caecums et sa vessie qui, pourvue de diverticules, atteint la ventouse orale. Cependant sa ressemblance avec $P$. donacis est assez frappante. Les renseignements que nous possédons à son sujet étant assez restreints, les éléments de comparaison sont peu nombreux. En dehors de la nature du premier hôte intermédiaire (Donax variabilis), $P$. donacis est caractérisé par un rapport ventousaire plus important $(2,7)$; ses caecums sont de taille plus réduite, l'orifice génital est placé plus près du bord antérieur de la ventouse ventrale et des auricules n'ont été vues que chez quelques spécimens. La différence la plus importante réside dans le fait que les métacercaires de l'espèce américaine sont d'âges divers, ce que je n'ai jamais observé chez $P$. timondavidi. En effet les divers organes et notamment ceux du système génital sont au même degré d'évolution.

L'adulte obtenu expérimentalement ne s'apparente à aucune des espèces déjà connues du genre Parvatrema. Il s'en distingue d'abord par sa très grande taille. Si l'on met à part $P$. ovoplenum (Jameson et Nicoll, 1913) Stunkard et Uzmann, 1958, dont on ne possède qu'une diagnose restreinte, l'espèce mytilicole est seule à posséder, avec 
$P$. borinqueñae, une unique glande vitelline; chez tous les autres, il y a deux masses vitellines distinctes. En outre, les auricules sur la ventouse orale ne sont présentes que chez $P$. borinqueñae, $P$. borealis et $P$. obscurum (Ching, 1960) James, 1964. Chez cette dernière espèce, l'orifice génital est contigu à la ventouse ventrale et chez $P$. borealis l'utérus remplit la presque totalité du corps. Chez $P$. borinqueñae, la ventouse orale n'est pas terminale; le rapport ventousaire et celui de la longueur du corps sur celle de la ventouse orale sont différents; la vessie excrétrice a une forme différente de celle de $P$. timondavidi.

Bien que l'espèce provençale soit nettement individualisée, il faut noter sa ressemblance, au niveau de la métacercaire avec $P$. donacis et à celui de l'adulte expérimental avec $P$. borinqueñae ; ceci permet d'envisager le genre Parvatrema comme très homogène.

\section{Considérations écologiques}

Les pourcentages d'infestation dans mes différentes stations sont les suivants :

Côte Sud de 1'Ile Riou : 0 \%.

Cap Croisette : $35 \%$.

Endoume : $60 \%$.

On peut interpréter ces variations en tenant compte de la notion d'épidémiotope proposée par J. Rebecq (1964). Deux cas sont à envisager en tenant compte du fait que l'on ignore le cycle de développement de $P$. timondavidi. De toutes façons, l'absence de la métacercaire de $P$. timondavidi dans les Moules de Riou montre que la station n'est pas un épidémiotope pour l'espèce ; cette absence incite à penser que le développement nécessite deux hôtes intermédiaires, le premier faisant défaut dans cette station. S'il était montré au contraire, que le développement nécessite un seul hôte intermédiaire (Mytilus galloprovincialis), on pourrait dire que l'hôte définitif naturel ne fréquente pas la station de l'île Riou.

Quant aux différences dans le taux d'infestation entre les Moules de la station du Cap Croisette et celles de la station d'Endoume, elles semblent pouvoir résulter de l'aspect topographique différent, la station du Cap Croisette étant plus « ouverte » que celle d'Endoume. Cette dernière représente un meilleur épidémiotope que celui du Cap Croisette. Dans les deux éventualités du cycle (soit un, soit deux hôtes intermédiaires), ces deux stations sont fréquentées par l'hôte définitif naturel et par le premier hôte intermédiaire s'il existe.

A la station d'Endoume, le nombre de Moules parasitées varie beaucoup en fonction de leur taille. La contamination maximum s'observe chez celles dont la longueur se situe entre $5 \mathrm{~cm}$ et $6 \mathrm{~cm}$. (fig. 6). Zelikman (1962) note que les Moules (M. edulis) de grande taille, c'est-à-dire celles servant de nourriture aux hôtes définitifs (Eider), sont les plus fréquemment parasitées. Mais comment expliquer cette décroissance de l'infestation chez les $M$. galloprovincialis de grande taille ? On peut penser que la longévité moyenne des métacercaires est telle qu'elles meurent chez les Moules les plus âgées, d'où leur raréfaction. D'ailleurs, ce fait est corroboré par l'observation suivante : le nombre maximum de métacercaires est trouvé dans les Moules de $3 \mathrm{~cm}$ à $4 \mathrm{~cm}$ (78); 
ce nombre diminue ensuite en fonction de la taille : 54 dans les Moules de $4 \mathrm{~cm}$ à $5 \mathrm{~cm}$ et 36 pour celles de $5 \mathrm{~cm}$ à $6 \mathrm{~cm}$.

Les recherches que je poursuis pour trouver les sporocystes et les cercaires de $P$. timondavidi n'ont point encore abouti malgré le très grand nombre de $M$. galloprovincialis disséqués. Dans l'éventualité où il existerait un premier hôte intermédiaire, j'ai recherché ces stades dans de nombreux Mollusques appartenant à la biocénose des Moules ou dans la proximité de ces dernières. C'est ainsi que j'ai examiné, mais sans résultats, divers Lamellibranches: Brachydontes minimus Poli, Lima squamosa Lmck, Lasoea rubra Montagu et plusieurs espèces de Prosobranches des genres Littorina, Monodonta, Patella et Diodora.

Mais les Moules peuvent être aussi contaminées directement par les métacercaires. En effet, Zelikman a observé des métacercaires libres de $G$. choledochus rampant

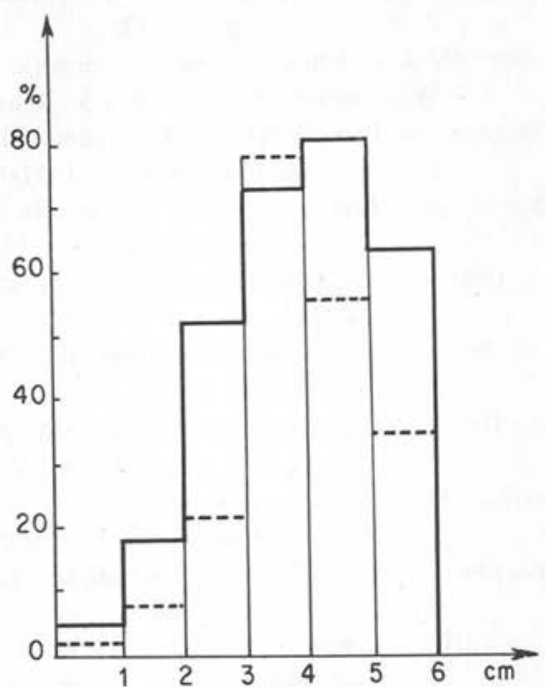

FIG. 6. - Variation de l'infestation chez les Moules d'Endoume. En traits pleins le taux d'infestation. En pointillé le nombre maximum de métacercaires observées sur le substrat ou au voisinage des siphons des Moules. Leur sortie vers le milieu extérieur permettrait selon l'auteur la contamination de Moule à Moule. Personnellement j'ai observé la sortie des métacercaires de G. strigatus Lebour des Cardium edule Linné ; cependant ceci ne se produit jamais pour $P$. timondavidi. En effet, j'ai isolé dans de petits cristallisoirs de nombreuses Moules et jamais je n'ai vu des métacercaires en sortir ; d'ailleurs, comment pourraient-elles le faire avec leur kyste mucilagineux? A plusieurs reprises j'ai transplanté des métacercaires dans des Moules indemnes provenant de stations non infestées; je ne les ai jamais retrouvées. La durée de vie des métacercaires dans l'eau de mer ne dépasse jamais un à deux jours.

En conclusion, pour $P$. timondavidi, il semble qu'il n'y a pas de possibilité d'infestation de Moule à Moule par les métacercaires.

Les résultats de ces observations morphologiques, biologiques et écologiques qui m'ont amené à donner une description détaillée de Parvatrema timondavidi, espèce bien individualisée, restent à compléter en ce qui concerne le cycle de développement par les voies tant de l'expérimentation que de l'écologie.

(Laboratoire de Zoologie, Faculté des Sciences de Marseille)

\section{Bibliographie}

Bartoli (P.), 1963. - Note préliminaire sur l'anatomie et la biologie de Parvatrema timondavidi n. sp. (Trematoda, Digenea). C.R. Acad. Sc. Paris, 273, p. 518-520. 
CABle (R. M.), 1953. - The life cycle of Parvatrema borinqueñae gen. and sp. nov. (Trematoda: Digenea) and the systematic position of the subfamily Gymnophallinae. J. Parasit., 39, p. 408-421.

CHIng (H. L.), 1960. - Some digenetic trematodes of shore birds at Friday Harbour, Washington. Proc. helm. soc. Wash., 27, p. 53-62.

Dollfus (R.-Ph.), 1923. - Le Trématode des perles de nacre des moules de Provence. C.R. Ac. Sc. Paris, 176, p. 1427-1429.

Dubors (R.), 1901. - Sur le mécanisme de la formation des perles fines dans le Mytilus edulis. C.R. Acad. Sc. Paris, 133, p. 603-605.

-, 1903. - L'origine des perles chez le Mytilus galloprovincialis. C.R. Acad. Sc. Paris, 136, p. 178-179.

-, 1907. - Sur les métamorphoses du Distome parasite des Mytilus perliers. C.R. Soc. Biol. Paris, 63, p. 334-336.

-, 1909. - Contribution à l'étude des perles fines, de la nacre, et des animaux qui les produisent. Ann. Univ. Lyon (N. S.), 29.

GIARD (A.), 1907. - Sur les Trématodes margaritigènes du Pas-de-Calais (Gymnophallus somateriae Levinsen et G. bursicola Odhner). C.R. Soc. Biol. Paris, 63, p. 416-420.

HopkINS (S. H.), 1958. - Trematodes parasites of Donax variabilis at Mustang Island, Texas. Institute of Marine Science, 5, p. 301-311.

JAmes (B. L.), 1964. - The life cycle of Parvatrema homoeotecnum sp. nov. (Trematoda : Digenea) and a review of the family Gymnophallidae Morozov, 1955. Parasitology, 54 , p. 1-41.

JAMeSON (H. L.), 1902. - On the origin of pearls. Proc. Zool. Soc. London, 1, p. 140-166.

- et Nicoll (W.), 1913. - On some parasites of the Scoter duck (Oidemia nigra) and their Relation to the Pearl-including Trematode in the Edible Mussel. Proc. Zool. Soc. London, 1, p. 53-63.

Palombi (A.), 1924. - Le cercarie del genere Gymnophallus dei Mitili. Publ. Staz. Zool. Napoli, 5, (2), p. 137-152.

-, 1934. - Gli stadi larvali dei Trematodi del Golfo di Napoli. $1^{\circ}$ Contributo allo studio della morfologia, biologia e sistematica delle cercarie marine. Publ. Staz. Zool. Napoli, $14, \mathrm{n}^{\circ} 1$, p. 51-94.

-, 1940. - Gli stadi larvali dei trematodi del Golfo di Napoli. Riv. Parassitol., 4, $\mathrm{n}^{\circ}$ 1, p. 7-30.

REBECQ (J.), 1964. - Recherches systématiques, biologiques et écologiques sur les formes larvaires de quelques Trématodes de Camargue. Thèse Marseille, $\mathrm{n}^{\circ}$ d'ordre 122, 223 p., pl. h.t.

SinitZINE (D. T.), 1911. - Les générations parthénogénétiques des Trématodes et leurs descendants dans les Mollusques de la Mer Noire. (En Russe). Mém. Acad. imp. Sc. Saint-Pétersbourg, $8^{e}$ série, Classe physico-mathématique, $30, \mathrm{n}^{\circ} 5,127 \mathrm{p}$.

Stunkard (H. W.) et Uzmann (J. R.), 1958. - Studies on digenetic Trematodes of the genera Gymnophallus and Parvatrema. Biol. Bull., 115, $\mathrm{n}^{\circ}$ 2, p. 276-302.

SzIDAT (L.), 1958. - Ueber eine ungewöhnliche form parthenogenetischer Vermehrung bei metacercarien einer Gymnophallus-Art aus Mytilus platensis, Gymnophallus australis n. sp. des südatlantik. Z. Parasitenkunde, 22, p. 196-213.

Zelikman (E. A.), 1962. - Les larves de Trématodes (famille des Gymnophallidae Morozov, 1955 (Trematoda: Digenea) et leur développement. (En Russe). Trudy murmansk. morsk. biol. Inst., 4, p. 186-201. 